\title{
Selective light trapping of plasmonic stack metamaterials by circuit design
}

\author{
Jinfeng Zhu, ${ }^{* a}$ Lirong Zhang, ${ }^{a}$ Shan Jiang, ${ }^{a}$ Jun-Yu Ou ${ }^{b}$ and Qing Huo Liu ${ }^{c}$
}

\begin{abstract}
Plasmonic metamaterials have wide applications on light trapping and manipulation. However, most of their design typically rely on solving Maxwell's equations via computational electromagnetics, which is time-consuming and limits the design flexibility. Here, we combine the transmission line circuit theory to design the plasmonic stack metamaterials in the nearinfrared range. In virtue of the simplicity and high efficiency of circuit theory, we design various light trapping functions by using plasmonic stack metamaterials, including comb filtering, short pass, long pass, band pass and band stop. Our study reveals the field-circuit relationship for the light-matter interaction of nanostructure stacks and provides a powerful method for the quick design of functional plasmonic metamaterials.
\end{abstract}

\section{Introduction}

Plasmonic metamaterials are artificial and engineered materials in combination with the extraordinary optical properties from surface plasmons and subwavelength nanostructured building units. $^{1,2}$ These optical properties stem from the unique structure of the metal-dielectric composites with features smaller than the wavelength of light. ${ }^{3}$ Spatial light waves hitting such a metamaterial are transformed into surface plasmons, which are shorter in wavelength than the incident light and show local field enhancement. ${ }^{4}$ Plasmonic metamaterials have wide applications on light energy trapping, subwavelength microscopy, optical computing, optical cloaking, biomedical and chemical sensing. ${ }^{5-9}$ In the optical range, a lot of plasmonic metamaterials have been reported such as reconfigurable plasmonic metamaterials, ${ }^{10,11}$ plasmonic metamaterial absorbers, ${ }^{12,13}$ low-loss plasmonic metamaterials, ${ }^{14,15}$ and hyperbolic metamaterials. ${ }^{16,17}$ In most of these research efforts, the plasmonic metamaterials are typically designed by solving Maxwell's equations based on the classic electromagnetic wave theory. In virtue of the great advances in computational electromagnetics, various plasmonic metamaterials can be systematically designed and optimized by the full wave electromagnetic simulation. However, the use of full wave simulation method usually demands on high computation cost and a lot of time, and this seriously limits the flexible and smart structural design for plasmonic metamaterials. A concept of optical circuit might provide an alternative and promising approach to facilitate the quick design of plasmonic metamaterials, ${ }^{18-20}$ because it allows powerful simplification and effective modularization for the function of each lumped element, which has been extensively and successfully adopted in the RF and microwave range.

Even though the circuit concept might give a new opportunity for the convenient design of plasmonic metamaterials in the optical range, many equivalent schemes used in the RF and microwave range cannot be directly transplanted due to the significant differences between the electromagnetic properties of component materials in the circuit for these two spectral ranges. Up to date, an efficient circuit design approach for plasmonic metamaterials with various optical functions is still quite in demand. In this study, we propose a scenario based on the optical transmission line circuit theory, and effectively assume the optical nanostructures as lumped elements for further design and analysis. By using this method in the near-infrared range, the various plasmonic metamaterials are designed with a series of filter circuit functions such as comb filtering, band pass, band stop, short pass and long pass. Our method has the capability to customize the optical functions simply and efficiently like that in the RF and microwave domains, and shows promising potential in the design of plasmonic metamaterials.

\section{Methods}

In this study, we develop a model of equivalent circuit based on the classical transmission line theory, which is used to guide the design of plasmonic metamaterials in the near-infrared range. We start from extracting the equivalent circuit of the simplest metal/dielectric/metal (MDM) subwavelength component with two nanostructured gold blocks and a nanoscale air gap between them, as shown in Fig. 1. The permittivity of bulk gold in the near-infrared range is described by the Drude model with the plasmon frequency $\omega_{p}=1.21 \times 10^{16} \mathrm{rad} / \mathrm{s}$ and the damping constant $\gamma=1.34 \times 10^{14} \mathrm{rad} / \mathrm{s} .{ }^{21,22}$ The free space plane wave with $p$-polarization from the top of the nanostructure is used to excite the gap plasmonic mode. All the gold nanostructure blocks used in our investigations have the thickness larger than the skin depth $\delta$ in order to exclude the coupling effects between the upper and lower surfaces of each gold block. The adjacent nanostructures of unit cells for the metamaterial are also assume to be far enough from each other in order to exclude their electromagnetic coupling. The subwavelength component is assumed as an equivalent circuit with a series of RLC lumped elements, as shown in Fig. 1. In this circuit, the lumped elements include the ohmic resistance $R$, the kinetic inductance $L_{e}$ induced by the drifting electrons within the skin depth of gold, the Faraday inductance $L_{m}$ due to the mutual effects between the two gold blocks, the equivalent capacitance $C$ for the gap between the two gold blocks and the capacitance $C_{\text {air }}$ outside of the gap. They are represented as below, ${ }^{23,24}$

$$
\begin{gathered}
R=\frac{2 \gamma w}{\varepsilon_{0} \delta l \omega_{p}^{2}} \\
L_{e}=\frac{2 w}{\varepsilon_{0} \delta l \omega_{p}^{2}} \\
L_{m}=\frac{\mu_{0} d w}{l}
\end{gathered}
$$




$$
\begin{gathered}
C=\frac{\alpha \varepsilon_{r} \varepsilon_{0} w l}{d} \\
C_{\text {air }} \approx 0
\end{gathered}
$$

where $\varepsilon_{0}$ and $\mu_{0}$ denote the permittivity and permeability of vacuum, respectively; $\varepsilon_{r}$ represents the relative permittivity of the material in the gap; $d, w$ and $l$ denote the thickness of the gap (not less than $2 \mathrm{~nm}$ ), the width and length of the blocks, respectively. To simplify the metamaterial modelling, the direction of $l$ is assume to be infinite for two dimensional light wave simulation, and we just adopt a unit length of $200 \mathrm{~nm}$ for circuit analysis. $\delta$ is about $24.5 \mathrm{~nm}$ for gold in the near-infrared range, ${ }^{22}$ and $C_{\text {air }}$ is close to $0 . \alpha$ is the tuning factor of the equivalent capacitance for the gap, and it is 0.122 for the air gap and will vary as the gap configuration changes. In the equivalent circuit, the plane wave excitation is assumed as the broadband voltage source $V_{0} e^{j \omega t}$, and the input impedance induced by the nanoscale gap can be calculated as below,

$$
Z_{i n}=R+j \omega\left(L_{e}+L_{m}\right)-\frac{j}{\omega C}
$$

where $\omega$ is the angular frequency of light waves. At the bottom of the nanostructure, the free space is assumed as the broadband perfect matched terminal without any reflection. On the resonance condition, the circuit impedance is perfectly matched and the imaginary part of $Z_{\text {in }}$ equals to 0 . Thus, we obtain the wavelength of plasmonic resonance as below,

$$
\lambda_{0}=2 \pi c \sqrt{\left(L_{e}+L_{m}\right) C}
$$

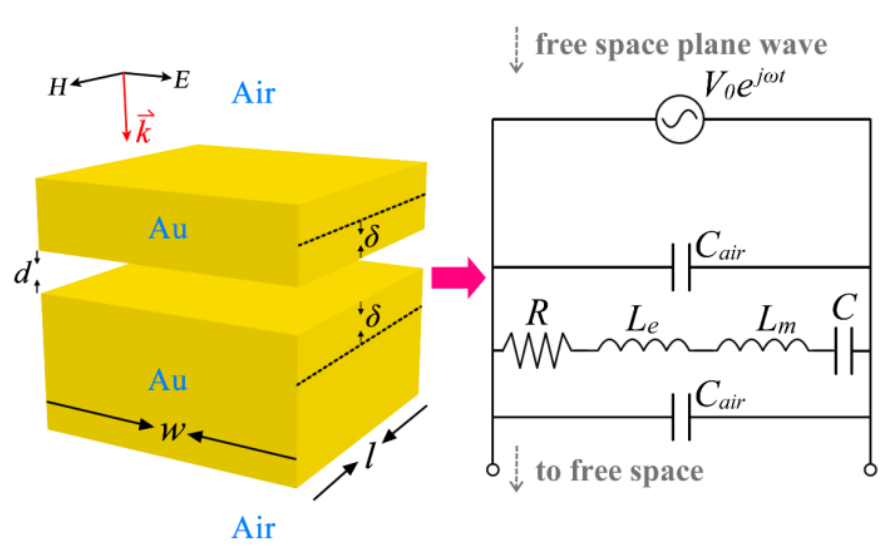

Fig. 1. A metamaterial unit cell of subwavelength component with two gold nanostructure blocks, where the symbols $d, w, l$ and $\delta$ denote the thickness of the gap, the width and length of the blocks, and the skin depth, respectively.

where $c$ is the vacuum light speed. The current in the $R L C$ branch is calculated as below,

$$
I=\frac{j \omega C V_{0}}{1+j \omega C R-\omega^{2}\left(L_{e}+L_{m}\right) C}
$$

It is worth mentioning that the ohmic loss is corresponding to the optical loss, and the circuit parameter $|I|^{2}$ is proportional to the optical absorbance in the spectrum.

The software Advanced Design System is also adopted for the equivalent circuit analysis of the plasmonic stack metamaterials. ${ }^{25}$ The software Comsol Multiphysics is used for the full wave simulation based on the finite element method (FEM). ${ }^{26}$ In the optical simulation, the adaptive inhomogeneous tetrahedral meshing is used to discretize the subwavelength unit cell, and the essential wave ports with Floquet periodic boundary conditions are adopted. The minimum edge length of the mesh element is as small as $0.05 \mathrm{~nm}$ to ensure the simulation convergence and reproducibility.

\section{Results and discussion}

\section{Circuit properties for the MDM unit of plasmonic metamaterials}

As shown in Fig. 2(a), the analytical circuit calculation and ADS (Advanced Design System) circuit simulation show very good consistency with the full wave simulation, which demonstrates the accuracy and feasibility of the proposed circuit method for the design of plasmonic stack metamaterials. According to Equation (7), the plasmonic resonance wavelength depends on the values of $L_{e}, L_{m}$ and $C$, which is determined by the nanostructure dimensions of $d, w$ and $l$. As shown in the absorbance spectra of Fig. 2(b), the resonance wavelength is increased from $1368 \mathrm{~nm}$ to $2205 \mathrm{~nm}$ as the thickness $d$ is reduced from $6 \mathrm{~nm}$ to $2 \mathrm{~nm}$, but it is proportionally increased from $1471 \mathrm{~nm}$ to $1764 \mathrm{~nm}$ as the width $w$ rises from $180 \mathrm{~nm}$ to $220 \mathrm{~nm}$. In Fig. 2(c) and Fig. 2(d), the results by the circuit calculation show that the resonance wavelength reduces with $d$ getting larger and increases with $w$ becoming larger, which demonstrates good agreement with the full wave simulation and validates the effectiveness of using the equivalent circuit (a)

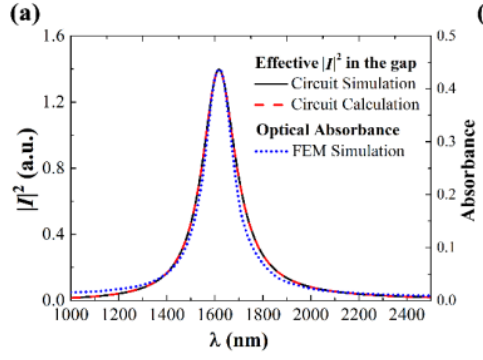

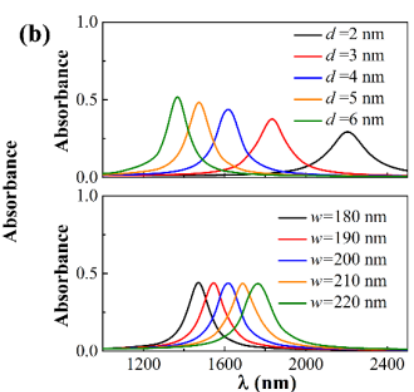

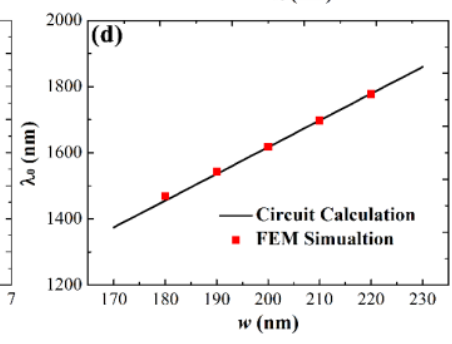

Fig. 2. (a) Optical reflectance and $|I|^{2}$ for a plasmonic gap, where $d=4 \mathrm{~nm}, w=200 \mathrm{~nm}$, the metamaterial period is $500 \mathrm{~nm}$, and the thicknesses of the upper and lower gold blocks are $50 \mathrm{~nm}$ and $100 \mathrm{~nm}$, respectively. (b) Optical absorbance as functions of the gap thickness and width. (c) Plasmonic resonance wavelength as a function of $d$. (d) Plasmonic resonance wavelength as a function of $w$.

with plasmonic lumped elements. Such circuit method illuminates the promising potential of quick design for plasmonic stack metamaterials, since it dramatically reduces the design time compared with the full wave simulation.

Equivalent plasmonic parallel circuits 
We next investigate the plasmonic metamaterial configuration with two stacked MDM nanostructures in a unit, as shown in Fig. 3(a). An infinite gold thin film with a thickness larger than $\delta$ is adopted as the bottom layer to block the optical transmission. In this structure, each MDM sandwich structure can be assumed as an RLC series circuit branch including the ohmic resistance, the kinetic inductance and Faraday inductance, and the equivalent capacitance for the corresponding dielectric gap. The base gold layer can be assumed as an inductance with the value much larger than other equivalent inductances, which reflects the input light wave signal. The air capacitance, the RLC circuits for both MDM sandwiches and the base inductance are paralleled in the entire circuit, as shown in Fig. 3(a). By optimizing the circuit parameters (i.e. the structural parameters) for each plasmonic gap based on Equation (1)-(4), we can obtain two separate absorption bands, which correspond to two block bands for the reflectance spectrum. The calculated values by circuit analysis show good agreement with the FEM simulation, as observed in Fig. 3(b). The optical field distributions are shown in Fig. 3(c), and they illuminate that there are two gap plasmon modes with near field enhancement within the upper alumina layer $(\lambda=1618 \mathrm{~nm})$ and the lower alumina layer $(\lambda=2033 \mathrm{~nm})$, respectively. For a gap plasmon mode, the corresponding magnetic field is guided in the alumina dielectric layer and along the direction vertical to the incident plane, and there is an electric dipole resonance in the gap leading to the strong ohmic loss in the metal. Such optical loss can be reflected by the ohmic
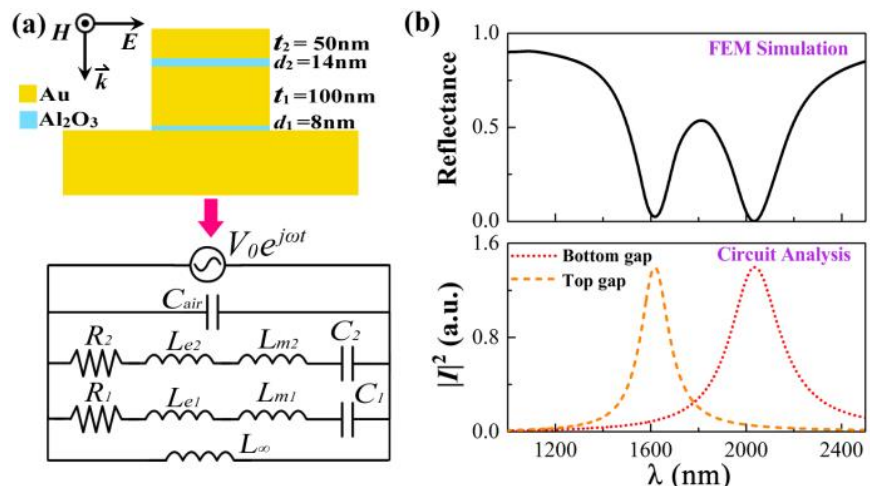

(c)

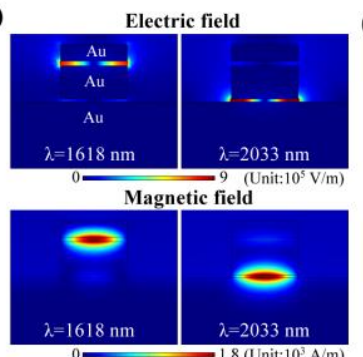

(d)

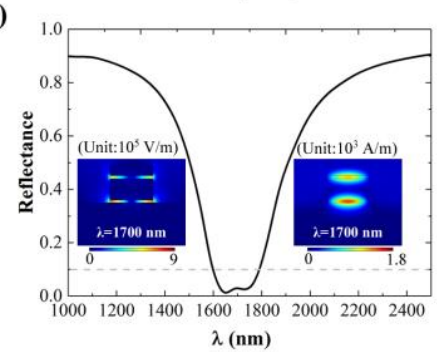

Fig. 3. (a) A subwavelength component with two stacked $\mathrm{Au} / \mathrm{Al}_{2} \mathrm{O}_{3} / \mathrm{Au}$ nanostructures, where the period is $500 \mathrm{~nm}$ and the structural parameters $d_{1}, t_{1}, d_{2}$ and $t_{2}$ are optimized for two stop bands. (b) Optical reflectance and effective $|I|^{2}$ for each plasmonic gap by the circuit analysis. (c) The corresponding electric and magnetic field distributions for the two plasmonic resonance wavelengths. (d) Optical reflectance as a function of wavelength when $d_{1}$ is changed to $11.5 \mathrm{~nm}$, and the insets are the corresponding electric and magnetic field distributions at $\lambda=1700 \mathrm{~nm}$.

resistance $R_{1}$ and $R_{2}$ as shown in the equivalent circuit of Fig. 3(a). Moreover, based on the circuit design for two parallel RLC branches, we can tune the lumped elements by changing the structural parameters for a customizable optical spectrum. For instance, when $d_{1}$ in Fig. $3(\mathrm{a})$ is changed from $8 \mathrm{~nm}$ to $11.5 \mathrm{~nm}$, the two separate stop bands by the two $\mathrm{Au} / \mathrm{Al}_{2} \mathrm{O}_{3} / \mathrm{Au}$ gaps can be merged into one stop band as shown in Fig. 3(d), which corresponds to a combined absorption band with the absorbance higher than $90 \%$ in the range from $1599 \mathrm{~nm}$ to 1790 $\mathrm{nm}$. The electric and magnetic field distributions in Fig. 3(d) indicate that the combined absorption is attributed to the adjacent plasmonic resonance wavelengths of the two $\mathrm{Au} / \mathrm{Al}_{2} \mathrm{O}_{3} /$ Au gaps.

Furthermore, we study the structure with three stacked MDM sandwich nanostructures with the corresponding equivalent circuit, as shown in Fig. 4(a). The circuit is similar to the one in Fig. 3(a), and it has three parallel RLC branches with a very large base inductance to block the optical transmission. By optimizing the circuit parameters (i.e. the nanostructure parameters), three separate perfect absorption bands can be obtained, which correspond to three block bands (i.e. comb filtering feature) in the reflectance spectrum. The circuit analysis shows good consistency with the full wave FEM simulation, as shown in Fig. 4(b). The electric and magnetic field distributions are shown in Fig. 4(c), and they illuminate that there are three gap plasmon modes with near field enhancement within the upper alumina layer $(\lambda=1323 \mathrm{~nm})$, the middle alumina layer $(\lambda=1658 \mathrm{~nm})$ and the lower alumina layer $(\lambda=2145 \mathrm{~nm})$, respectively. Similar to the case in Fig. 3(c), for
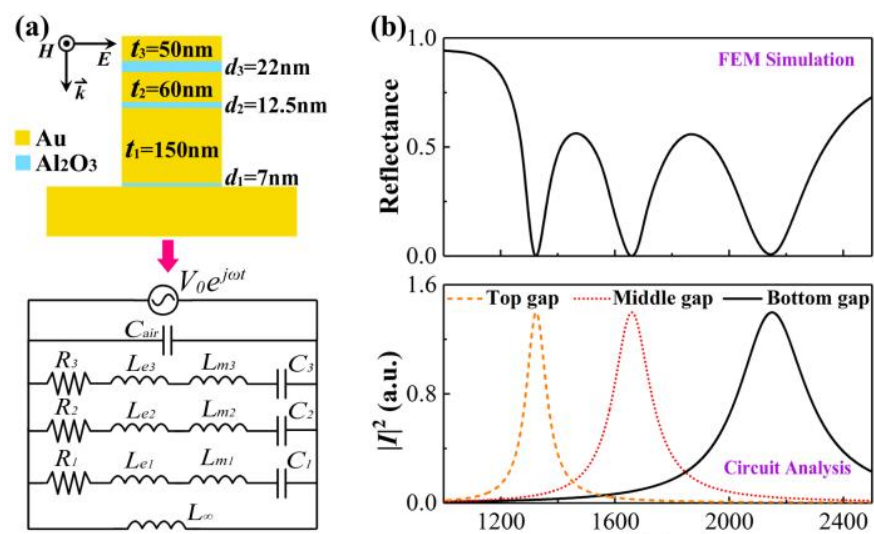

(c)
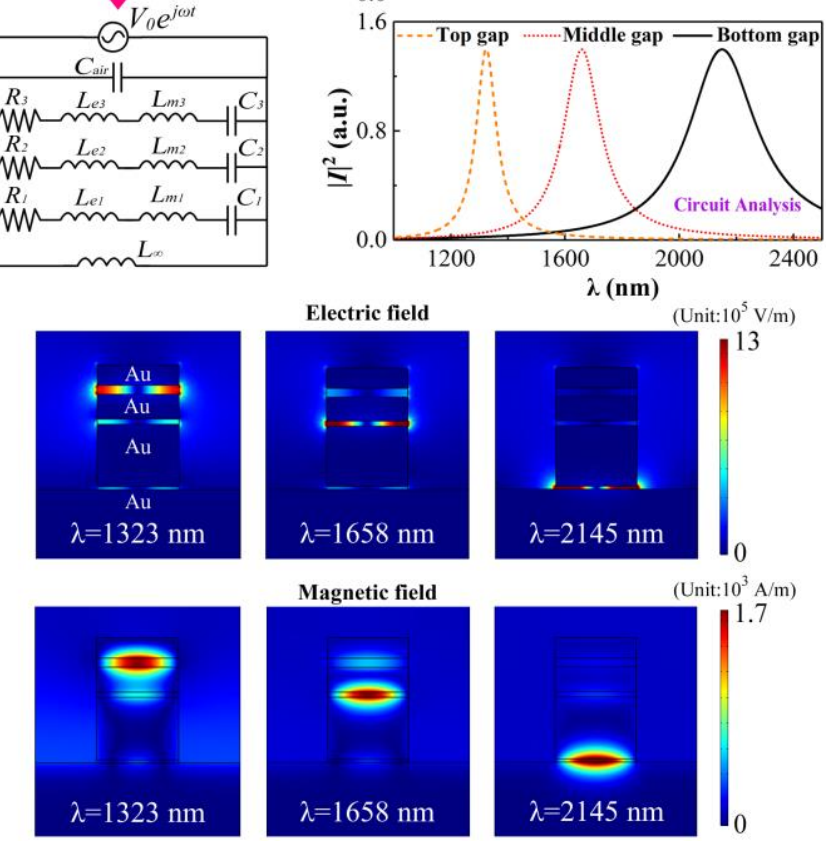

Magnetic field
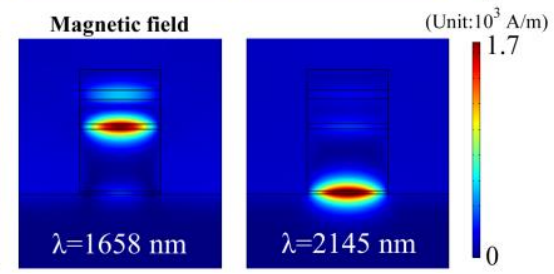

Fig. 4. (a) A subwavelength component with three stacked $\mathrm{Au} / \mathrm{Al}_{2} \mathrm{O}_{3} / \mathrm{Au}$ nanostructures, where the period is $500 \mathrm{~nm}$ and the structural parameters $d_{1}, t_{1}, d_{2}, t_{2}, d_{3}$ and $t_{3}$ are optimized for three stop bands. (b) Optical reflectance and $|I|^{2}$ for each plasmonic gap 
by the circuit analysis. (c) The corresponding electric and magnetic field distributions for the three plasmonic resonance wavelengths.

each gap plasmon mode in Fig. 4(c), the magnetic field is guided along the direction vertical to the incident plane, and the electric dipole resonance in the alumina gap results in high ohmic loss in gold and leads to perfect optical absorption.

\section{Functional plasmonic filter metamaterials for light trapping}

The transmission line circuit theory deduced from Fig. 1 to Fig. 4 provides the important design guide for other functional plasmonic stack metamaterials by using the full wave simulation. Based on this theory, one can also design four kinds of basic filter circuits, including short pass, long pass, band pass and band stop for various applications. Here, we design four plasmonic metamaterial filters with the above four circuit functions, as shown in the simulated results of Fig. 5. Since the optical absorption band for each MDM sandwich layer is determined by the wavelength of plasmonic resonance, we can assemble the MDM sandwich layers in order to tune the light filtering functions of these plasmonic metamaterials.

For the design of long pass plasmonic filter metamaterial, we use the subwavelength unit with 5 pairs of $\mathrm{Al}_{2} \mathrm{O}_{3} / \mathrm{Au}$ layered nanostructures with $w=200 \mathrm{~nm}$ and the period $P=500 \mathrm{~nm}$, which are on top a continuous gold layer of $200 \mathrm{~nm}$ thick. From the bottom $\mathrm{Al}_{2} \mathrm{O}_{3}$ layer to the top $\mathrm{Au}$ layer, the thicknesses are 19 $\mathrm{nm} / 160 \mathrm{~nm} / 22.5 \mathrm{~nm} / 160 \mathrm{~nm} / 24 \mathrm{~nm} / 100 \mathrm{~nm} / 26.5 \mathrm{~nm} / 100 \mathrm{~nm} /$
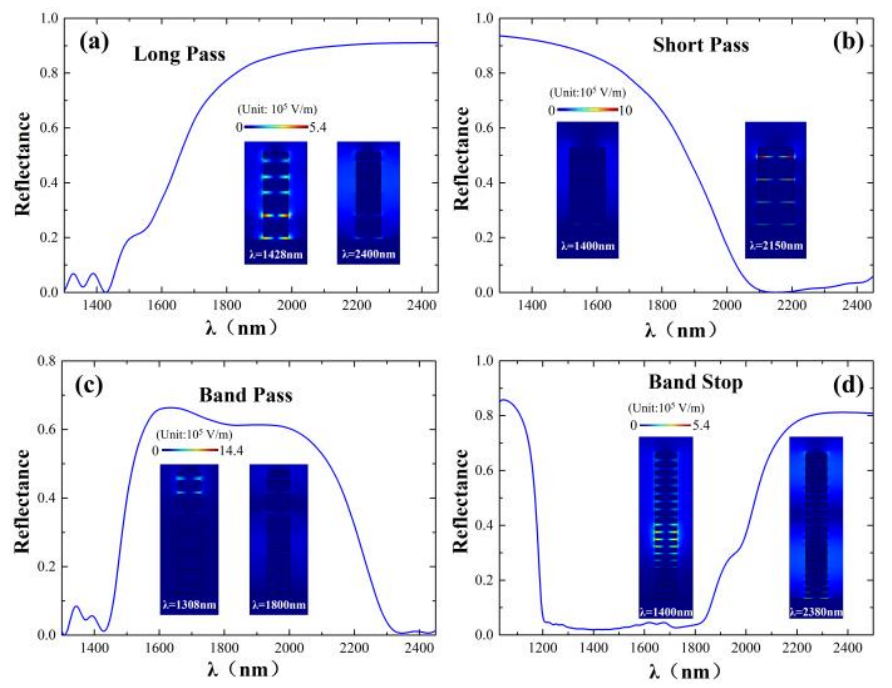

Fig. 5. Various plasmonic filter metamaterials. (a) Long pass. (b) Short pass. (c) Band pass (d) Band stop. Insets are the electric field distributions.

$31 \mathrm{~nm} / 50 \mathrm{~nm}$. Here, the thickness of $\mathrm{Al}_{2} \mathrm{O}_{3}$ is gradually increased from $19 \mathrm{~nm}$ to $31 \mathrm{~nm}$. These $\mathrm{Al}_{2} \mathrm{O}_{3}$ gaps confine the light energy in the short wavelength range, but they induce very weak lightmatter interaction in the long wavelength range, as observed in the electric field distributions of Fig. 5(a). When the wavelength is above $1800 \mathrm{~nm}$, the reflectance is higher than $80 \%$. However, the reflectance is lower than $7 \%$ and the light is trapped by all the MDM sandwich layers, when the wavelengths are below $1456 \mathrm{~nm}$. In contrast, we also design a short pass filter metamaterial, in which the subwavelength unit component with 4 pairs of $\mathrm{Al}_{2} \mathrm{O}_{3} / \mathrm{Au}$ stacked layers is used and the thicknesses from the bottom to the top are $5 \mathrm{~nm} / 120 \mathrm{~nm} / 5.5$ $\mathrm{nm} / 120 \mathrm{~nm} / 6 \mathrm{~nm} / 120 \mathrm{~nm} / 7 \mathrm{~nm} / 50 \mathrm{~nm}$. The corresponding reflectance spectrum is shown in Fig. $5(\mathrm{~b})$. The reflectance is higher than $80 \%$ when the wavelength is less than $1684 \mathrm{~nm}$, and it is lower than $10 \%$ as the wavelength becomes larger than $2026 \mathrm{~nm}$. The insets of electric field distributions in Fig. 5(b) demonstrate that the extremely weak light-matter interaction at the short wavelength of $\lambda=1400 \mathrm{~nm}$ and the near field enhancement in all the gaps leading to very high optical loss at the long wavelength of $\lambda=2150 \mathrm{~nm}$.

In order to design the band pass plasmonic filter metamaterial, we combine two assemblies of MDM stacked layers for the high absorption ratios at long wavelengths and short wavelengths, respectively, as shown in Fig. 5(c). The subwavelength unit has a continuous gold base layer of $200 \mathrm{~nm}$ thick and a period of $500 \mathrm{~nm}$. On top of the gold base layer, there are 8 alternating $\mathrm{Al}_{2} \mathrm{O}_{3} / \mathrm{Au}$ stacked nanostructures with the width of $200 \mathrm{~nm}$. From the bottom to the top, their thicknesses are $4.7 \mathrm{~nm} / 80 \mathrm{~nm} / 4.8 \mathrm{~nm} / 120 \mathrm{~nm} / 5.5 \mathrm{~nm} / 140$ $\mathrm{nm} / 19 \mathrm{~nm} / 160 \mathrm{~nm} / 22.5 \mathrm{~nm} / 160 \mathrm{~nm} / 24 \mathrm{~nm} / 160 \mathrm{~nm} / 26.5$ $\mathrm{nm} / 100 \mathrm{~nm} / 31 \mathrm{~nm} / 50 \mathrm{~nm}$. The first three MDM sandwich layers supply the near-unity absorption at the long wavelengths above $2300 \mathrm{~nm}$, while the other 5 MDM sandwich layers support the high absorption at the short wavelengths below $1430 \mathrm{~nm}$. As shown in Fig. 5(c), the reflectance ratios for these two wavelength ranges are below $7 \%$, whereas the reflectance is higher than $60 \%$ in the wavelength range from $1529 \mathrm{~nm}$ to 2045 $\mathrm{nm}$. The pass band is as wide as $516 \mathrm{~nm}$. On the contrary, we also design the band stop plasmonic filter metamaterial, in which an assembly of MDM sandwich layers is used for extremely high absorbance ( $>0.95$ ) at a very wide wavelength band from $1213 \mathrm{~nm}$ to $1830 \mathrm{~nm}$, as shown in Fig. 5(d). The subwavelength unit has a continuous gold bottom layer of 200 $\mathrm{nm}$ thick with a period of $500 \mathrm{~nm}$. On top of the continuous gold layer, 20 pairs of $\mathrm{Al}_{2} \mathrm{O}_{3} / \mathrm{Au}$ layers are adopted. From the bottom to the top, all the Au layer have the thickness of $50 \mathrm{~nm}$, and the thicknesses of 20 layers of $\mathrm{Al}_{2} \mathrm{O}_{3}$ are an arithmetic sequence from $9 \mathrm{~nm}$ to $35.6 \mathrm{~nm}$ with the thickness difference of $1.4 \mathrm{~nm}$ between adjacent $\mathrm{Al}_{2} \mathrm{O}_{3}$ layers. As observed in the insets of Fig. $5(d)$, the broadband near-unity absorption is attributed to the plasmonic resonance modes with the successive wavelengths in all the dielectric gaps.

The above study in Fig. 5 only refers to the change of stack layer thickness for tuning the optical response, and it typically requires the fabrication process using many times of deposition for metal and dielectric layers associated with nanolithography, which is complicated in practice. In order to reduce the complexity, we take account of the stack width as another degree of design freedom and introduce two stack nanostructure components with different widths in a subwavelength unit $(P=1000 \mathrm{~nm})$, as shown in the simulated result of Fig. 6(a). This stack nanostructure only adopts 2 pairs of $\mathrm{Al}_{2} \mathrm{O}_{3} / \mathrm{Au}$ layers, but it supports a broadband high optical 
absorbance (>0.91) in the wavelength range from $1471 \mathrm{~nm}$ to $1935 \mathrm{~nm}$. Such metamaterial configuration significantly facilitates the nanofabrication in process. The high optical absorption results from four combined plasmonic resonances at the wavelengths of $1505 \mathrm{~nm}, 1625 \mathrm{~nm}, 1758 \mathrm{~nm}$ and $1893 \mathrm{~nm}$, respectively, which are observed as the electric and magnetic field distributions in Fig. 6(b) and Fig. 6(c). Due to the optimized

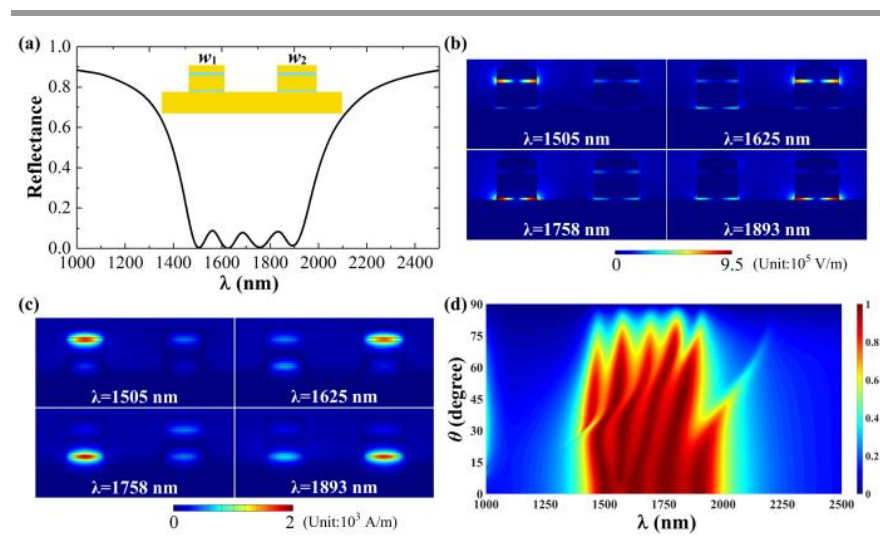

Fig. 6. (a) Broad stop band by using two subwavelength stacked nanostructures in a unit, where $w_{1}=200 \mathrm{~nm}, w_{2}=220 \mathrm{~nm}$ and the $\mathrm{Al}_{2} \mathrm{O}_{3} / \mathrm{Au} / \mathrm{Al}_{2} \mathrm{O}_{3} / \mathrm{Au}$ layer thicknesses from the bottom to the top are $12 \mathrm{~nm} / 120 \mathrm{~nm} / 18 \mathrm{~nm} / 50 \mathrm{~nm}$. (b) The corresponding electric field distributions for the four plasmonic resonance wavelengths. (c) The corresponding magnetic field distributions for the four plasmonic resonance wavelengths. (d) Optical absorbance as a function of wavelength and incident angle, where the color bar denotes absorbance ratio.

thickness and width for each $\mathrm{Al}_{2} \mathrm{O}_{3}$ gap, there are four different plasmonic excitation modes within the gaps to support the broadband optical absorption. We further investigate the absorption dependence on the incident angle, as can be seen in Fig. 6(d). It indicates that the broadband absorption is kept for a large range of incident angles, which is very similar to many other regular MDM absorbers, ${ }^{27-29}$ and would also enable many new applications on light trapping and manipulation.

\section{Conclusions}

In summary, we combine the transmission line circuit theory with the full wave simulation to design functional plasmonic stack metamaterials in the near-infrared range. Functional plasmonic stack metamaterials with various light-matter interactions are achieved by the guide of circuit analysis. Our method sufficiently gets use of the advantages of the circuit design and provides a fast way to explore new plasmonic metamaterials for wide applications on subwavelength light trapping and manipulation.

\section{Conflicts of interest}

The authors declare that there are no conflicts of interest related to this article.

\section{Acknowledgements}

This work was supported by NSAF (Grant No. U1830116), Fujian Provincial Department of Science and Technology (Grant No. 2017J01123), the Fundamental Research Funds for the Central
Universities (Grant No. 20720190010), and the fund of Key Laboratory of THz Technology, Ministry of Education, China.

\section{References}

1 A. Boltasseva and H. A. Atwater, Science, 2011, 331, 290-291.

2 Y. Cai, J. Zhu and Q. H. Liu, Appl. Phys. Lett., 2015, 106, 043105.

3 J. Zhu, M. Xue, H. Shen, Z. Wu, S. Kim, J.-J. Ho, A. HassaniAfshar, B. Zeng and K. L Wang, Appl. Phys. Lett., 2011, 98, 151110.

4 Y. Xu, Y. Fu and H. Chen, Nat. Rev. Mater., 2016, 1, 16067.

5 B. Liu, S. Chen, J. Zhang, X. Yao, J. Zhong, H. Lin, T. Huang, Z. Yang, J. Zhu, S. Liu, C. Lienau, L. Wang and B. Ren, Adv. Mater., 2018, 30, 1706031.

6 K. Zhao, H. Zhang, Y. Fu and S. Zhu, Nanophotonics, 2018, 7 339-345.

7 T. Zhu, Y. Zhou, Y. Lou, H. Ye, M. Qiu, Z. Ruan and S. Fan, Nat. Commun., 2017, 8, 15391.

8 H. Chen, C. T. Chan and P. Sheng, Nat. Mater., 2010, 9, $387-$ 396.

9 K. V. Sreekanth, Y. Alapan, M. ElKabbash, E Ilker, M. Hinczewski, U. A. Gurkan, A. D. Luca and G. Strangi, Nat. Mater., 2016, 15, 621-627.

10 J.-Y. Ou, E. Plum, J. Zhang and N. I. Zheludev, Nat. Nanotech. 2013, 8, 252-255.

11 J.-Y. Ou, E. Plum, J. Zhang and N. I. Zheludev, Adv. Mater. 2015, 28, 729-733.

12 Y. Cai, J. Zhu, Q. H. Liu, T. Lin, J. Zhou, L. Ye and Z. Cai, Opt. Express, 2015, 23, 32318-32328.

13 J. Zhu, L. Zhang, Y. Bai, H. Liu, N. Feng, J. Zhou, B. Zeng, T. Lin and Q. H. Liu, IEEE Photonic. Tech. L., 2017, 29, 504-506.

14 G. V. Naik, J. Liu, A. V. Kildishev, V. M. Shalaev and A. Boltasseva, P. Natl. Acad. Sci. USA, 2012, 109, 8834-8838.

15 J. D. Caldwell, L. Lindsay, V. Giannini, I. Vurgaftman, T. L. Reinecke, S. A. Maier and O. J. Glembocki, Nanophotonics 2015, 4, 44-68.

16 A. Poddubny, I. Iorsh, P. Belov and Y. Kivshar, Nat. Photonics, 2013, 7, 948-957.

17 K. V. Sreekanth, Y. Alapan, M. ElKabbash, E. Ilker, M. Hinczewski, U. A. Gurkan, A. De Luca and G. Strangi, Nat. Materials, 2016, 15, 621-627.

18 N. Engheta, Science, 2007, 317, 1698-1702.

19 F. Benz, B. de Nijs, C. Tserkezis, R. Chikkaraddy, D. O. Sigle, L. Pukenas, S. D. Evans, J. Aizpurua and J. J. Baumberg, Opt. Express, 2015, 23, 33255-33269.

20 T. J. Davis, D. E. Gómez and A. Roberts, Nanophotonics, 2017, 6, 543-559.

21 S. A. Maier, Plasmonics: Fundamentals and Applications, Springer, New, York, 2007.

22 E. D. Palik, Handbook of Optical Constants of Solids, Academic, San Diego, 1998.

23 T. W. Hughes and S. Fan, Nano Lett., 2016, 16, 5764-5769.

24 B. J. Lee, L. P. Wang and Z. M. Zhang, Opt. Express, 2008, 16, 11328-11336.

25 https://www.keysight.com/

26 J. Zhou, F. Tao, J. Zhu, S. Lin, Z. Wang, X. Wang, J.-Y. Ou, Y. Li, Q. H. Liu, Nanophotonics, 2019, 8, 307-316.

27 J. Hao, J. Wang, X. Liu, W. J. Padilla, L. Zhou and M. Qiu, Appl. Phys. Lett., 2010, 96, 251104.

28 Y. Cai, Z. Wang, S. Yan, L. Ye and J. Zhu, Opt. Mater. Express, 2018, 8, 3295-3306.

29 L. Lei, S. Li, H. Huang, K. Tao and P Xu, Opt. Express, 2018, 26, 5686-5693. 